\title{
Endothelial activation and apoptosis mediated by neutrophil-dependent interleukin 6 trans-signalling: a novel target for systemic sclerosis?
}

\author{
Theresa C Barnes, ${ }^{1}$ David G Spiller, ${ }^{2}$ Marina E Anderson, ${ }^{1}$ Steven W Edwards, ${ }^{2}$ \\ Robert J Moots ${ }^{1}$
}

- Additional tables and figures are published online only. To view these files please visit the journal online at (http://ard. bmi.com).

${ }^{1}$ School of Clinical Sciences, University of Liverpool, Liverpool, UK

${ }^{2}$ School of Biological Sciences, University of Liverpool, Liverpool, UK

\section{Correspondence to \\ Professor Robert J Moots, Academic Rheumatology, Clinical Sciences Centre, University Hospital Aintree, Longmoor Lane, Liverpool L9 7AL, UK: r.j.moots@liv.ac.uk}

SWE and RJM contributed equally to this work

Accepted 16 September 2010 Published Online First 10 November 2010

\begin{abstract}
Objectives Systemic sclerosis (SSc) is a connective tissue disease associated with significant morbidity and mortality and generally inadequate treatment. Endothelial cell activation and apoptosis are thought to be pivotal in the pathogenesis of this disease, but the mechanisms that mediate this remain unknown.

Methods Human dermal microvascular endothelial cells were cultured with healthy control neutrophils in the presence of $25 \%$ healthy control or SSc serum for $24 \mathrm{~h}$. Apoptosis was measured by annexin V-FITC binding and endothelial cell activation was measured using an allophycocyanin-conjugated E-selectin antibody. Fluorescence was quantified and localised using confocal microscopy.
\end{abstract}

Results SSc serum resulted in significantly increased apoptosis $(p=0.006)$ and E-selectin expression $(p=0.00004)$ in endothelial cells compared with control serum, effects that were critically dependent on the presence of neutrophils. Recombinant interleukin 6 (IL-6) reproduced these findings. Immunodepletion of IL- 6 and the use of an IL-6 neutralising antibody decreased the effect of SSc serum on E-selectin expression. Soluble gp130, which specifically blocks IL-6 trans-signalling, negated the effect of SSc serum on both E-selectin expression and apoptosis.

Conclusions SSc serum induces endothelial cell activation and apoptosis in endothelial cell-neutrophil co-cultures, mediated largely by IL-6 and dependent on the presence of neutrophils. Together with other pathologically relevant effects of IL-6, these data justify further exploration of IL-6 as a therapeutic target in SSc.

\section{INTRODUCTION}

Systemic sclerosis (SSc) is a multisystem connective tissue disease characterised by fibrosis of the skin and internal organs and by microvascular injury. There is considerable morbidity and a significant increase in mortality. ${ }^{1}$ Despite recent developments, current treatments remain inadequate and therefore there is a continuing need for additional therapeutic strategies.

Endothelial cell activation and apoptosis are thought to be pivotal in the pathogenesis of SSc. Some evidence points to an increase in endothelial cell apoptosis, although there is a lack of in vivo evidence to corroborate this. ${ }^{2}$ The University of California at Davis line 200 chicken, an animal model of SSc, consistently exhibits endothelial cell apoptosis in skin and internal organs from serial tissue samples, preceding mononuclear cell infiltrate and development of fibrosis. ${ }^{3} 4$
Markers of endothelial cell activation, including an increase in expression of cell adhesion molecules, may be observed by immunohistochemical examination of lesional tissue samples from patients with SSc. An increase in the serum levels of soluble adhesion molecules including soluble intercellular adhesion molecule 1 (ICAM-1) and soluble E-selectin are found in SSc patients compared with controls, and these correlate with tissue expression of endothelial adhesion molecules and severity of disease manifestations. ${ }^{5-7}$

Interleukin 6 (IL-6) is a pleiotropic cytokine that is increased in the serum of patients with SSc and correlates with markers of disease activity. ${ }^{8-12}$ Immunocytochemistry demonstrates an increase in the levels of IL-6 in the lesional skin of patients with SSc and this is associated with the late stages of the disease. ${ }^{13}$ IL- 6 has many functions that may be relevant to the pathogenesis of SSc including endothelial cell activation. ${ }^{14}$

Neutrophils were shown by Hussein et a l15 $^{15}$ to be increased in lesional biopsies of patients with SSc compared with controls. Others have explored neutrophil function in SSc, in particular their ability to contribute to oxidative stress by the production of reactive oxygen species. The data are contradictory and are largely limited by old-fashioned neutrophil isolation procedures which can lead to neutrophil activation. ${ }^{16}{ }^{17} \mathrm{~A}$ recent study has, however, shown that neutrophils produce less reactive oxygen species in vitro than control neutrophils when unstimulated. ${ }^{18}$ In agreement with this, we have found that neutrophils from patients with SSc are hypofunctional in tests of reactive oxygen species generation and chemotaxis (unpublished data). This may reflect in vivo stimulation and hence in vitro 'exhaustion'. Proteomic studies show that SSc neutrophils have increased expression of proteins that are also increased on stimulation with lipopolysaccharide or tumour necrosis factor (TNF), again indicative of neutrophil activation in vivo (unpublished data).

Activated neutrophils have the potential to release agents capable of endothelial injury, including reactive oxygen species and proteases, and the ability to affect cytokine signalling. In order to explore whether neutrophils could have a role in endothelial cell injury in SSc, the purpose of this study was to determine the effects of SSc serum on neutrophils and their interaction with endothelial cells in vitro. These experiments reveal a role for IL- 6 in induction of endothelial cell activation online under the BMJ Journals unlocked scheme, see http:// ard.bmj.com/info/unlocked.dtl 
and apoptosis in SSc, and highlight this cytokine as a potential therapeutic target.

\section{METHODS}

The study was approved by the Sefton local ethics committee in accordance with the Helsinki declaration. Informed written consent was taken from patients with $\mathrm{SSc}^{19}$ and from healthy volunteers.

\section{Materials}

The following materials were used in the study: human dermal microvascular endothelial cells (HDMECs; Promocell, Heidelberg, Germany), recombinant IL-6, soluble gp130 (sgp130; R\&D, Minneapolis, Minnesota, USA), direct immunodepletion kit (Thermo, Waltham, Massachusetts, USA), polyclonal rabbit antihuman recombinant IL-6 (rIL-6) antibody (ab6672; Abcam, Cambridge, UK), Polymorphprep (Axis-Shield, Dundee, UK), Annexin V-FITC, mitotracker red (Invitrogen, Paisley, California, USA), allophycocyanin (APC)-conjugated mouse monoclonal antihuman E-selectin antibody (ab51241; Abcam), calcein-AM (Calbiochem, San Diego, California, USA).

\section{Culture of HDMECs}

HDMECs were cultured in 48-well plates in microvascular endothelial cell medium (Promocell). Cultures were used in passage 4-8 when they reached $90 \%$ confluence. Under these conditions, cell phenotype is guaranteed up to passage 14 (Promocell).

\section{Isolation of neutrophils}

Neutrophils were isolated from healthy control peripheral blood using Polymorphprep as described in the manufacturer's instructions. Contaminating erythrocytes were removed using ammonium chloride lysis buffer $\left(\mathrm{KHCO}_{3} 3.4 \mathrm{mM}, \mathrm{NH}_{4} \mathrm{Cl} 155 \mathrm{mM}\right.$, EDTA $96.7 \mu \mathrm{M})$. Neutrophils were routinely examined for purity using morphological analysis of cytospins; purity was $>95 \%$ immediately after isolation. Neutrophils were resuspended in microvascular endothelial cell medium supplemented with $2 \mathrm{mM}$ glutamine at a concentration of $10^{6}$ cells $/ \mathrm{ml}$.

\section{Preparation of serum samples}

Serum samples were collected in clot activating tubes (Vacutainer). Serum was aspirated and flash frozen in liquid nitrogen, then filtered $(0.4 \mu \mathrm{m}$ filter) to remove particulate material that could interfere with confocal microscopy.

\section{Preparation of endothelial cell:neutrophil co-cultures}

Endothelial cell culture medium was replaced with $450 \mu \mathrm{l}$ fresh medium or neutrophil suspension and $150 \mu \mathrm{l} \mathrm{SSc}$ or healthy control serum. $1.5 \mu \mathrm{l}$ annexin $\mathrm{V}$ and $2.5 \mu \mathrm{l}$ of a 1:10 dilution of the E-selectin antibody were added to each well. Co-cultures were incubated for $24 \mathrm{~h}$ at $37^{\circ} \mathrm{C}$ in a $5 \% \quad \mathrm{CO}_{2}$ humidified atmosphere.

\section{$24 \mathrm{~h}$ time lapse imaging}

This is outlined in the online supplement.

\section{Confocal microscopy}

Confocal images were taken using a LSM-710 (Zeiss Welwyn Garden City, Hertfordshire, UK) confocal microscope using the Zen 2009 software. Annexin V-FITC was excited using the $488 \mathrm{~nm}$ laser whereas APC was excited using the $633 \mathrm{~nm}$ laser.
Images were taken using the optimum separation of emission spectra mode. Duplicate images were taken for each experimental variable. A z-stack of 13 images was taken over a distance of $9.91 \mu \mathrm{m}$, focusing from the endothelial cells to the neutrophils. Images were taken using a $10 \times$ fluar 0.5 NA objective.

Images were analysed using Image J software (NIH, USA). Images representing the maximal fluorescence values for red and green fluorescence were measured for total fluorescence intensity. Values were corrected for fluorescence intensity of cells cultured in media alone.

\section{Serum cytokine profiling}

Serum concentrations of granulocyte colony-stimulating factor, interferon $\gamma$, granulocyte-macrophage colony-stimulating factor, TNFo, IL-1 $\beta$, IL-2, IL-4, IL-5, IL-6, IL-8, IL-10, IL-17 and IL-Ra were quantified using a 13-plex Luminex kit.

\section{Assessing the role of IL-6 in neutrophil:endothelial cell co-cultures}

Heat-inactivated pooled male AB serum was filtered $(0.4 \mu \mathrm{m}$ filter) and spiked with $200 \mathrm{pg} / \mathrm{ml}$ rIL-6. This was added to wells instead of SSc or control serum. The IL- 6 concentrations used reflected the levels of IL- 6 found in serum samples from patients with SSc by Luminex.

In order to assess whether IL- 6 played a role in the effect of SSc serum in neutrophil:endothelial cell co-cultures, three different experiments were performed. First, IL- 6 was removed from SSc serum using a direct immunodepletion kit in accordance with the manufacturer's instructions and $60 \%$ immunodepletion was confirmed by western blotting (data not shown). Second, the polyclonal anti-IL-6 antibody was added to wells at a concentration of 1:400 to neutralise IL- 6 in the co-culture. Finally, sgp130, an inhibitor of IL- 6 trans-signalling, was added to the wells at a concentration of $500 \mathrm{ng} / \mathrm{ml}$.

\section{Data analysis}

Normality of the data was assessed using SPSS. E-selectin data were found to be normally distributed and therefore differences between means were analysed using the paired t test. Annexin $\mathrm{V}$ staining was not normally distributed and therefore differences were analysed using the Wilcoxon signed rank test. The strength and significance of clinical correlations were described using the Spearman correlation coefficient.

\section{RESULTS}

Patient characteristics are summarised in table 1 in the online supplement.

\section{Effect of SSc serum on apoptosis and E-selectin expression in endothelial cell:neutrophil co-cultures}

$\mathrm{SSc}$ serum resulted in greater annexin-V binding $(\mathrm{p}=0.006)$ and E-selectin $(p=0.00004)$ expression than control serum when added to endothelial cell:neutrophil co-cultures (figure 1). When corrected for background staining in serum-free media, control serum did not increase E-selectin expression whereas SSc serum had a 2.5-fold increase in E-selectin expression. Control serum had a 2.5-fold increase in annexin-V staining whereas SSc serum had a sevenfold increase compared with media alone. There was wide variation in the effects of control serum and, in particular, SSc serum (figure 2).

Annexin-V binding was detected on both neutrophils and endothelial cells. E-selectin is a specific endothelial cell marker and reflects endothelial cell activation. However, E-selectin 


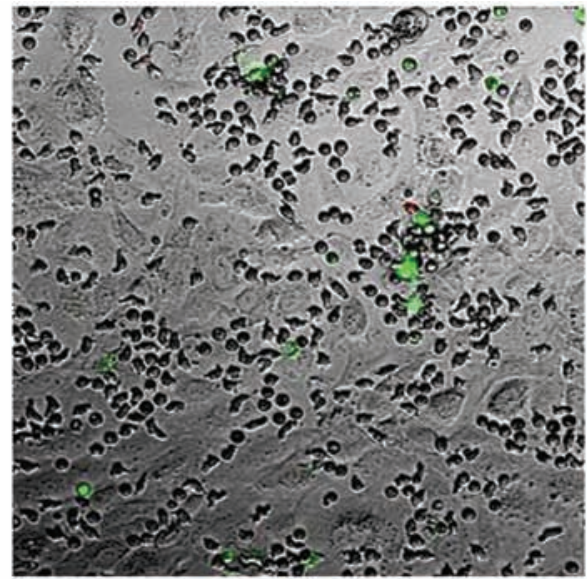

(A)

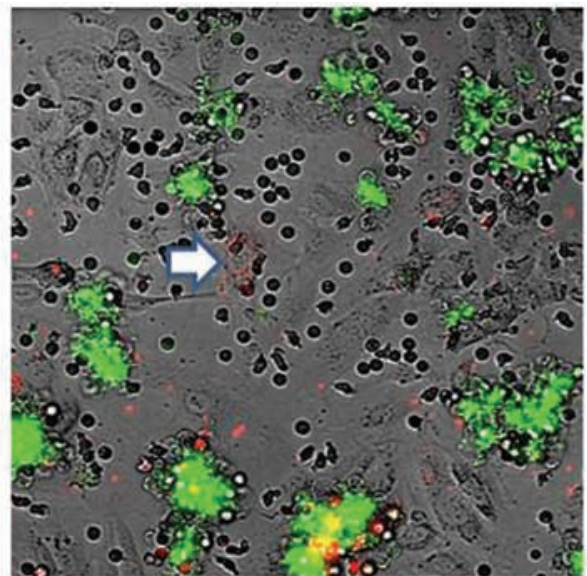

(B)

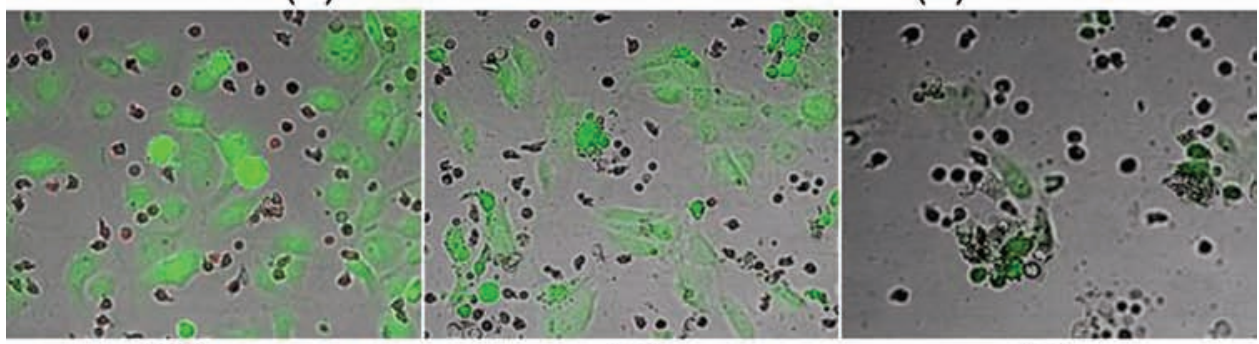

(C)

(D)

(E)

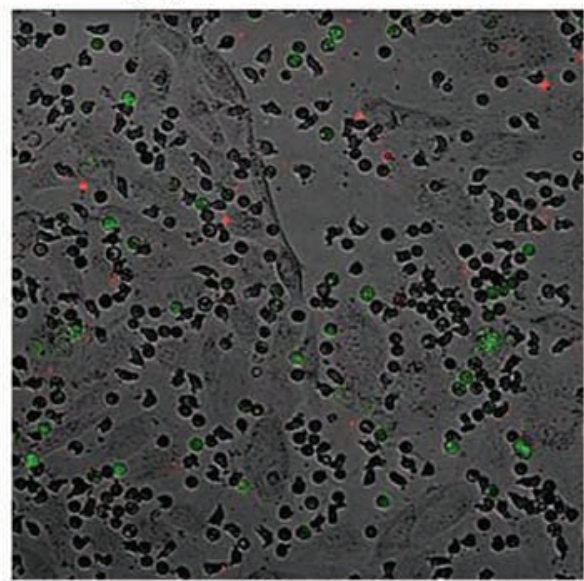

(F)

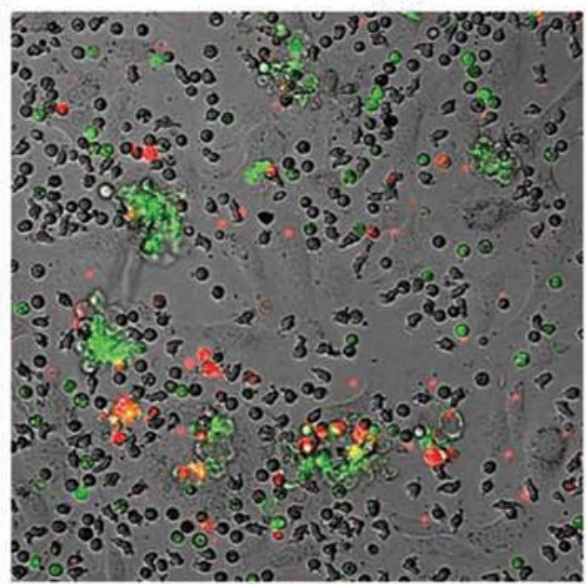

(G)

Figure 1 Systemic sclerosis (SSc) serum increases endothelial activation in neutrophil co-cultures. Human dermal microvascular endothelial cells were co-cultured with healthy control neutrophils for $24 \mathrm{~h}$ in the presence of $25 \%$ healthy control or SSc serum. Apoptosis was measured using annexin V-FITC (green) and E-selectin expression was measured using an allophycocyanin (APC)-conjugated monoclonal antibody (red). Confocal images of the effects of $(A)$ normal serum or $(B)$ SSc serum on the co-cultures show that the SSc serum promotes aggregation and enhanced apoptosis and E-selection expression ( $p=0.006$ and 0.00004 , respectively, $n=17)$. The arrow indicates endothelial cell $E$-selectin expression. (C), (D) and (E) are images taken from time lapse movies of neutrophil:endothelial cell co-cultures in the presence of $25 \%$ SSc serum. Neutrophils were labelled with mitotracker (red) and endothelial cells were labelled with calcein-AM (green). (C) shows a time zero image, (D) shows an image taken after $1 \mathrm{~h}$ which shows endothelial cell blebbing and detachment (indicative of apoptosis) and (E) shows that the cellular aggregates comprise both endothelial cells (green) and neutrophils. Neutrophil:endothelial cell co-cultures labelled with annexin V-FITC (green) and an APC-conjugated monoclonal anti-E-selectin antibody (red) cultured for $24 \mathrm{~h}$ with (F) $25 \%$ AB serum or (G) $25 \%$ AB serum containing recombinant interleukin 6 $(200 \mathrm{pg} / \mathrm{ml})$.

expression in co-cultures was predominantly associated with cellular aggregates where it was difficult to distinguish morphologically between neutrophils and endothelial cells.

High magnification z-stacks through the cellular aggregates were performed, but these could not unambiguously assign localised E-selectin staining to a particular cell type in these co-cultures. Cell-specific counterstaining was therefore used. 24-Hour time lapse image series of co-cultures, where endothelial cells were stained with calcein-AM and neutrophils with mitotracker red, showed that both endothelial cells and neutrophils were present in the cellular aggregates (figure 1E). Time lapse analyses also enabled us to show that endothelial cell apoptosis (as evidenced by membrane blebbing and cell detachment) was the initiating factor for the formation of the aggregates (figure 1D).

Single cell cultures were also performed as controls to determine if the effects on endothelial cell apoptosis and E-selectin expression required the presence of neutrophils. Spontaneous 

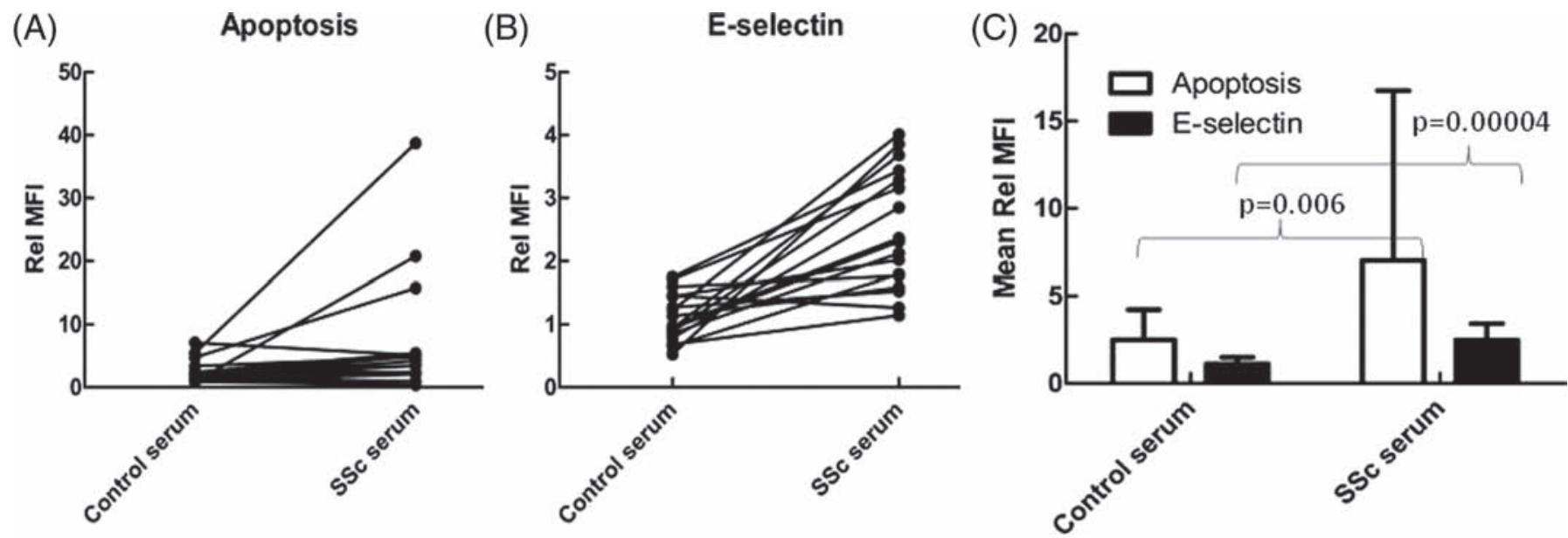

Figure 2 Systemic sclerosis (SSc) serum induces endothelial cell apoptosis and E-selectin expression in neutrophil co-cultures. (A) Changes in apoptosis and (B) changes in E-selectin expression of endothelial cells when incubated with individual control and SSc serum samples ( $n=17$ ). While there were marked variations in responses, particularly for SSc serum, both apoptosis and E-selectin expression were significantly enhanced after incubation with SSc serum compared with the effects of control serum. (C) Summary data shown as mean \pm SD values ( $n=17)$. Rel MFI, mean fluorescence intensity corrected to levels in media alone.

levels of apoptosis in endothelial cell cultures were negligible even after $24 \mathrm{~h}$. SSc serum did not lead to enhanced apoptosis or E-selectin expression in endothelial cell cultures compared with the effects of control serum (see figure 1 in online supplement). In neutrophil cultures alone there was some low-level background E-selectin expression (presumed to be non-specific binding), but there was no difference between control and SSc serum samples. There was no difference in neutrophil apoptosis when cultured with SSc serum or control serum in neutrophil only cultures (see figure 1 in online supplement).

E-selectin expression (figure 1G) was located in endothelial cells (indicated by the arrow), but most of the e-selectin expression appeared in small mobile bodies approximately 1-2 $\mu \mathrm{m}$ in diameter. These most likely represent endothelial microparticles which are generated by activated endothelial cells and are increased in the serum of patients with SSc. ${ }^{20}$ In addition, some staining was associated with what appear to be neutrophils. When supernatants were removed from the cultures after $24 \mathrm{~h}$ and read on the flow cytometer, there was some staining for E-selectin in the neutrophil fraction gated according to typical forward and side scatter but there was no difference between control and SSc serum (see figure 2 in online supplement).

No statistically significant differences were found in the effects on E-selectin expression and apoptosis in endothelial cell:neutrophil co-cultures between the serum of patients with limited and diffuse SSc. In addition, no significant difference was seen between serum from patients with early and late disease when early disease was defined as either $<12$ months or $<3$ years. Disease-modifying therapy did not make any significant difference to the effects of the serum.

\section{Effects of recombinant IL-6 on neutrophil:endothelial cell co-cultures}

Serum levels of IL-6 were increased in patients with SSc compared with controls. The IL-6 levels were consistent with those reported by Radstake et a ${ }^{21}$ in plasma samples from a large cohort of patients with SSc measured by Bioplex, which is similar to the Luminex kit used in this study. However, the levels were higher than those previously reported in SSc serum measured by ELISA in smaller cohorts. ${ }^{9}$
The IL-6 levels correlated significantly with the levels of endothelial activation $(\rho=0.829, p=0.042$ ) (measured by binding of a PE conjugated ICAM antibody) and apoptosis $(\rho=0.943$, $\mathrm{p}=0.005$ ) in neutrophil:endothelial cell co-cultures containing control or SSc serum (see figure 3 in online supplement).

The addition of rIL- 6 to endothelial cell cultures containing heat-inactivated $A B$ serum significantly increased annexin $V$ staining ( $p=0.008)$ and $\mathrm{E}$-selectin $(\mathrm{p}=0.006)$ expression, but only when neutrophils were present in the co-cultures (figure 3), reproducing the effects of SSc serum. Interestingly, co-cultures spiked with rIL-6 also induced the cell aggregates that were commonly seen in co-cultures with SSc serum (figure 1G).

\section{Role of IL-6 in the effect of SSc serum on neutrophil:endothelial cell co-cultures}

Immunodepletion of IL-6 from SSc serum resulted in a significant decrease in E-selectin expression in co-cultures $(p=0.01)$, with levels of expression similar to those seen with control serum. Immunodepletion of IL- 6 also decreased levels of apoptosis, but this did not reach statistical significance because of the large variation in the effects of SSc serum on apoptosis $(p=0.06$, figure 4$)$.

IL-6 neutralisation with a polyclonal anti-IL-6 antibody significantly abrogated the effect of SSc serum on E-selectin expression and apoptosis in co-cultures ( $p=0.005$ and $p=0.001$, respectively). Neutralising antibodies had no effect on apoptosis levels or E-selectin expression in co-cultures containing control serum (figure 5).

sgp130 acts as a decoy receptor, binding to sILR/IL- 6 complexes and preventing them from signalling through cellular gp130 receptor. It therefore specifically blocks trans-signalling but not signalling through the IL- 6 receptor. ${ }^{22}$ The addition of sgp130 reversed the effect of SSc on E-selectin expression $(p=0.033)$; in neutrophil-endothelial cell co-cultures there was a tendency for gp130 to decrease apoptosis but this did not reach statistical significance $(p=0.06)$, probably because of the high degree of variability and the small sample size (figure 6).

\section{DISCUSSION}

The data presented here shed important new light on the molecular mechanisms that underlie changes in endothelial 
cell function in SSc. We have shown that SSc serum induces endothelial cell apoptosis and E-selectin expression in vitro, but only in the presence of neutrophils. We also provide compelling evidence that IL- 6 in SSc serum is responsible for these functional changes and propose that IL- 6 trans-signalling, via neutrophil receptor shedding, is involved. These observations may thus open up new therapeutic strategies for the treatment of this disease.

These data, although important, do not confirm that IL-6 plays an important role in vivo. Further studies to confirm activation of IL- 6 trans-signalling pathways in lesional tissue would go some way to demonstrate this. In addition, the role of IL- 6

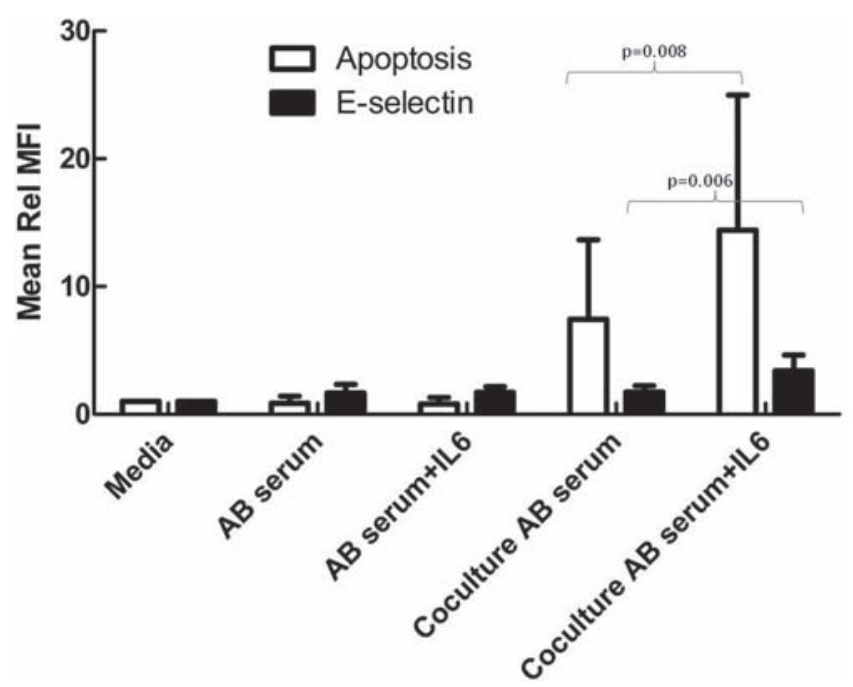

Figure 3 Addition of interleukin 6 (IL-6) to control serum mimics the effects of systemic sclerosis serum. Human dermal microvascular endothelial cells were cultured with and without neutrophils in the presence of $25 \%$ heat inactivated $A B$ serum for $24 \mathrm{~h}$. As indicated, some cultures also contained $200 \mathrm{pg} / \mathrm{ml}$ recombinant IL-6 (rlL-6). Apoptosis was measured using annexin V-FITC and E-selectin expression was measured using an allophycocyanin-conjugated monoclonal antibody. Mean fluorescence intensity (MFI) was measured by confocal microscopy and expressed as a fold change $( \pm S D, n=8)$ corrected to levels in media alone (Rel MFI). rlL-6 resulted in a significant increase in apoptosis and E-selectin expression, but only when neutrophils were present in the cultures. in vascular disease models of SSc-for example, the Fra-2 transgenic mouse ${ }^{23}$ - should also be explored, although it is important to note that differences in mouse and human immunology exist which could influence the outcome. ${ }^{24}$

Neutrophils express the IL-6 receptor (IL-6R) and may respond to IL-6, albeit weakly, through this signalling pathway. ${ }^{25}$ Endothelial cells do not express IL-6R and are only capable of responding to IL- 6 when this cytokine is bound to soluble IL-6R (sIL-6R); this complex then binds to the ubiquitously expressed gp130 receptor-that is, via trans-signalling. There are pools of sIL-6R, IL- 6 and sgp130 in the circulation, the latter capable of preventing trans-signalling. It is therefore the balance of all three of these elements that dictates whether IL-6 signalling occurs.

sIL-6R is generated by two separate processes: by proteolytic cleavage of membrane IL-6R or by synthesis and release of an alternatively spliced version of the receptor. ${ }^{26-29}$ Neutrophils are the dominant source of sIL-6R in the serum ${ }^{30} 31$ via proteolytic cleavage from the neutrophil membrane which can be stimulated by $C$ reactive protein, $\mathrm{f}-\mathrm{Met}$-Leu-Phe and chemokines such as IL-8, leukotriene $\mathrm{B}_{4}\left(\mathrm{LTB}_{4}\right)$ and platelet activating factor. $^{32}$ There is evidence to suggest that neutrophils do not express the alternatively spliced version of IL-6R. Interestingly, in addition to IL-6, we found raised concentrations of IL-8 in $\mathrm{SSc}$ serum (see table 2 in online supplement). This has been reported previously, as has an increase in IL-8 in lesional skin by immunocytochemistry associated with early disease. ${ }^{13} 33-35$ An increase in $\mathrm{LTB}_{4}$ has also been reported in the bronchoalveolar lavage fluid of patients with SSc with interstitial lung disease, and fibroblasts isolated from patients with SSc produce increased $\mathrm{LTB}_{4}$ when stimulated in vitro. ${ }^{36}{ }^{37} \mathrm{IL}-6 \mathrm{R}$ shedding also occurs when neutrophils undergo apoptosis via ADAM17. ${ }^{38}$

Endothelial cell activation is reported in response to IL-6 trans-signalling. ${ }^{14} 39$ Given that trans-signalling blockade with sgp130 abrogates the effects of SSc serum on endothelial cell activation in co-cultures, we hypothesise that enhancement of trans-signalling by local release of IL- $6 \mathrm{R}$ from neutrophils is responsible for this effect. However, these data do not exclude neutrophil activation by trans-signalling leading to further intermediates which are responsible for endothelial cell activation. Increased levels of sIL-6R and normal levels of sgp130 have previously been described in SSc serum. ${ }^{11}$
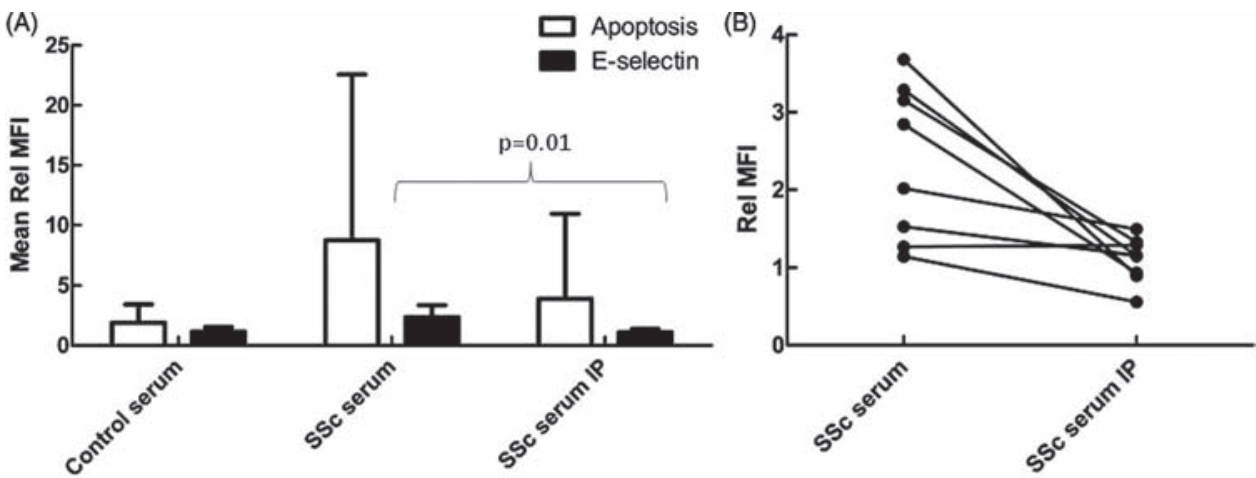

Figure 4 Interleukin 6 (IL-6) depletion from systemic sclerosis (SSc) serum decreases its effects on endothelial cell activation. Human dermal microvascular endothelial cells were cultured with healthy control neutrophils for $24 \mathrm{~h}$ in the presence of $25 \%$ healthy control, SSc serum or SSc serum immunodepleted of IL-6 using a direct immunodepletion kit (IP). Apoptosis was measured using annexin V-FITC and E-selectin expression was measured using an allophycocyanin-conjugated monoclonal antibody. (A) Mean fluorescence intensity (MFI) was measured by confocal microscopy and expressed as a fold change in mean fluorescence corrected to media alone (Rel MFI) $( \pm S D, n=8)$. (B) Changes in E-selectin expression in individual control and SSc pairs $(n=8)$. SSc serum resulted in significantly more E-selectin expression than control serum and this effect was abolished by the immunodepletion of IL-6 from the SSc serum. 

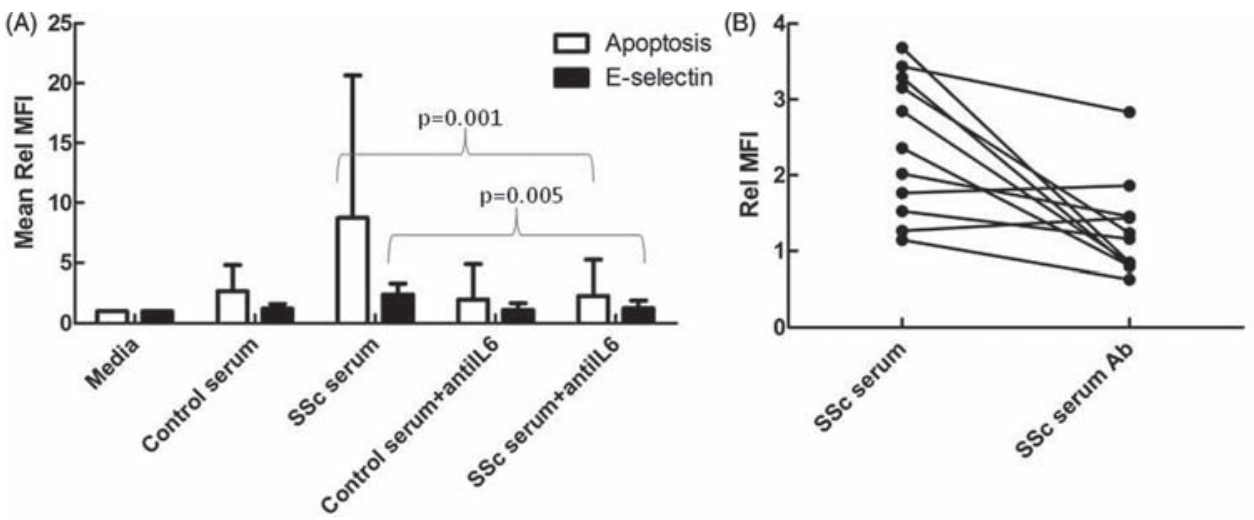

Figure 5 Neutralising interleukin 6 (IL-6) antibody decreases the effects of systemic sclerosis (SSc) serum on endothelial cell activation. Human dermal microvascular endothelial cells were cultured with healthy control neutrophils for $24 \mathrm{~h}$ in the presence of $25 \%$ healthy control or SSc serum. Neutralising IL-6 rabbit polyclonal antibody was added at a dilution of 1:400. Apoptosis was measured using annexin V-FITC and E-selectin expression was measured using an allophycocyanin-conjugated monoclonal antibody. (A) Mean fluorescence intensity (MFI) measured by confocal microscopy and expressed as a fold change in mean fluorescence corrected to media alone (Rel MFI) ( $\pm S D, n=11)$. (B) Changes in E-selectin expression in individual control and SSc pairs $(n=11)$. The addition of neutralising IL-6 antibodies to the co-cultures resulted in a decrease in the effect of SSc serum towards control levels.
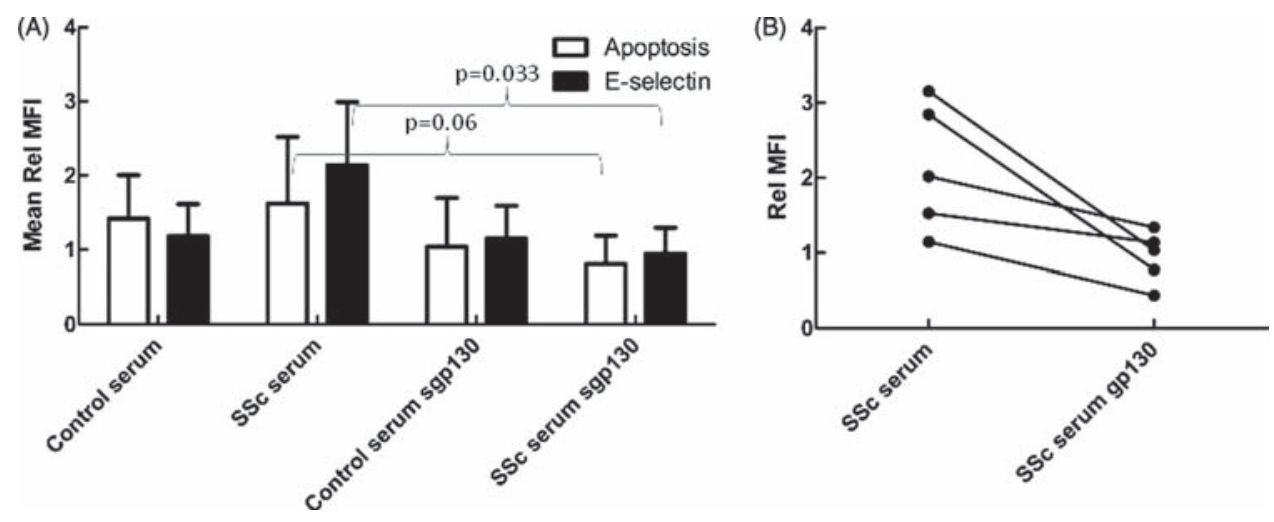

Figure 6 Soluble gp130 (sgp130) decreases the effects of systemic sclerosis (SSc) serum on endothelial cell activation. Human dermal microvascular endothelial cells were cultured with healthy control neutrophils for $24 \mathrm{~h}$ in the presence of $25 \%$ healthy control or SSc serum. sgp 130 was added ( $500 \mathrm{ng} / \mathrm{ml}$ ) to inhibit interleukin 6 trans-signalling. Apoptosis was measured using annexin V-FITC and E-selectin expression was measured using an allophycocyanin-conjugated monoclonal antibody. (A) Mean fluorescence intensity (MFI) measured by confocal microscopy and expressed as a fold change in mean fluorescence corrected to media alone (Rel MFI) $( \pm S D, n=5)$. (B) Changes in E-selectin expression in individual control and SSc pairs $(n=5)$. sgp130 significantly inhibited the effect of SSc serum on apoptosis and E-selectin expression.

IL-6 is an attractive therapeutic target in SSc. Increased levels of this cytokine occur in the serum of patients with SSc and correlate with disease activity. ${ }^{8-12}$ In addition, IL-6 is increased in histological specimens from the lesional skin of patients with SSc and is associated with late disease. ${ }^{13}$ IL- 6 is spontaneously secreted by monocytes and fibroblasts isolated from patients with SSc when cultured in vitro. ${ }^{40}{ }^{41}$ It is a pleiotropic cytokine with many roles that may be relevant to the pathogenesis of this disease.

IL-6 seems to act as a switch from acute to chronic inflammation. ${ }^{39} 4243$ It may also participate in the persistence of chronic inflammation as it stimulates fibroblasts and endothelial cells to secrete IL-6 which may then act in a paracrine fashion. ${ }^{39}$ It is important to point out that, although serum IL-6 levels are very heterogeneous in SSc, this does not mean that it does not play an important role in patients with relatively low serum levels as it is the local concentrations of IL- 6 at the cellular level which may be important, especially since there are pools of sIL-R and sgp130 in the circulation which can adsorb IL-6. The overall balance may depend on the lesional volume. This may explain why increased serum IL-6 concentrations are associated with the diffuse form of the disease and correlate with disease severity, and also why most patients with SSc do not exhibit the systemic effects of IL- 6 .

IL-6 has important effects on fibroblasts. It is a profibrotic cytokine that activates fibroblasts and results in an increase in collagen and glycosaminoglycan production. ${ }^{39}$ An IL-6 neutralising antibody was shown to decrease procollagen 1 production in cultured SSc fibroblasts. ${ }^{44}$

In conclusion, we hypothesise that IL- 6 plays a pivotal role in initiating and perpetuating the chronic inflammation, endothelial cell and fibroblast activation which are central to SSc. We have shown that SSc serum is capable of initiating endothelial cell apoptosis and activation in the presence of neutrophils. Neutrophils are the most abundant leucocytes in the circulation and, although they do not form a major part of the tissue infiltrate in SSc, they are constantly interacting with the endothelial surface. We propose that, through the donation of sIL-6R, they may play an important role in the pathogenesis of SSc.

Funding This work was funded by a Medical Research Council Clinical Training Fellowship held by TCB. The work was not in any part funded by interested parties in the pharmaceutical industry. The Aintree Arthritis Trust funded publication of this transcript. 


\section{Competing interests None.}

Ethics approval This study was conducted with the approval of the Sefton research ethics committee, Liverpool, UK.

Provenance and peer review Not commissioned; externally peer reviewed.

\section{REFERENCES}

1. Silman AJ. Scleroderma - demographics and survival. J Rheumatol Suppl 1997:48:58-61.

2. Fleming JN, Schwartz SM. The pathology of scleroderma vascular disease. Rheum Dis Clin North Am 2008:34:41-55.

3. Sgonc R, Gruschwitz MS, Dietrich H, et al. Endothelial cell apoptosis is a primary pathogenetic event underlying skin lesions in avian and human scleroderma. $J$ Clin Invest 1996;98:785-92.

4. Nguyen VA, Sgonc R, Dietrich $\mathrm{H}$, et al. Endothelial injury in internal organs of University of California at Davis line 200 (UCD 200) chickens, an animal model for systemic sclerosis (Scleroderma). J Autoimmun 2000;14:143-9.

5. Herrick AL, llingworth K, Blann A, et al. Von Willebrand factor, thrombomodulin, thromboxane, beta-thromboglobulin and markers of fibrinolysis in primary Raynaud's phenomenon and systemic sclerosis. Ann Rheum Dis 1996;55:122-7.

6. Blann AD, Herrick A, Jayson MI. Altered levels of soluble adhesion molecules in rheumatoid arthritis, vasculitis and systemic sclerosis. Br J Rheumatol 1995;34:814-19.

7. Gruschwitz MS, Hornstein OP, von Den Driesch P. Correlation of soluble adhesion molecules in the peripheral blood of scleroderma patients with their in situ expression and with disease activity. Arthritis Rheum 1995;38:184-9.

8. Matsushita T, Hasegawa M, Hamaguchi $Y$, et al. Longitudinal analysis of serum cytokine concentrations in systemic sclerosis: association of interleukin 12 elevation with spontaneous regression of skin sclerosis. J Rheumatol 2006;33:275-84.

9. Sato S, Hasegawa M, Takehara K. Serum levels of interleukin-6 and interleukin-10 correlate with total skin thickness score in patients with systemic sclerosis. J Dermatol Sci 2001:27:140-6.

10. Nagaoka T, Sato S, Hasegawa M, et al. Serum levels of soluble interleukin 6 receptor and soluble gp130 are elevated in patients with localized scleroderma. $J$ Rheumatol 2000;27:1917-21.

11. Hasegawa M, Sato S, Fujjmoto M, et al. Serum levels of interleukin 6 (IL-6), oncostatin M, soluble IL-6 receptor, and soluble gp130 in patients with systemic sclerosis. J Rheumatol 1998;25:308-13.

12. Stuart RA, Littlewood AJ, Maddison PJ, et al. Elevated serum interleukin-6 levels associated with active disease in systemic connective tissue disorders. Clin Exp Rheumatol 1995;13:17-22.

13. Koch AE, Kronfeld-Harrington LB, Szekanecz Z, et al. In situ expression of cytokines and cellular adhesion molecules in the skin of patients with systemic sclerosis. Their role in early and late disease. Pathobiology 1993;61:239-46.

14. Romano M, Sironi M, Toniatti C, et al. Role of IL-6 and its soluble receptor in induction of chemokines and leukocyte recruitment. Immunity 1997;6:315-25.

15. Hussein MR, Hassan HI, Hofny ER, et al. Alterations of mononuclear inflammatory cells, CD4/CD8 + T cells, interleukin 1beta, and tumour necrosis factor alpha in the bronchoalveolar lavage fluid, peripheral blood, and skin of patients with systemic sclerosis. J Clin Pathol 2005;58:178-84.

16. Maslen CL, Hall ND, Woolf AD, et al. Enhanced oxidative metabolism of neutrophils from patients with systemic sclerosis. Br J Rheumatol 1987;26:113-17.

17. Stevens TR, Hall ND, McHugh NJ, et al. Spontaneous neutrophil activation in patients with primary Raynaud's phenomenon and systemic sclerosis. Br J Rheumatol 1992;31:856.

18. Foerster J, Storch A, Fleischanderl S, et al. Neutrophil respiratory burst is decreased in scleroderma and normalized by near-infrared mediated hyperthermia. Clin Exp Dermatol 2006;31:799-806.

19. LeRoy EC, Medsger TA, Jr. Criteria for the classification of early systemic sclerosis. $J$ Rheumatol 2001;28:1573-6.

20. Guiducci S, Distler JH, Jüngel $\mathrm{A}$, et al. The relationship between plasma microparticles and disease manifestations in patients with systemic sclerosis. Arthritis Rheum 2008;58:2845-53.

21. Radstake TR, van Bonn L, Broen J, et al. The pronounced Th17 profile in systemic sclerosis (SSc) together with intracellular expression of TGF $\beta$ and IFN $\gamma$ distinguishes SSc phenotypes. PLoS One 2009;4:1-9.
22. Jones SA, Rose-John $\mathrm{S}$. The role of soluble receptors in cytokine biology: the agonistic properties of the sIL-6R/L-6 complex. Biochim Biophys Acta 2002;1592:251-63.

23. Maurer B, Busch N, Jüngel A, et al. Transcription factor fos-related antigen-2 induces progressive peripheral vasculopathy in mice closely resembling human systemic sclerosis. Circulation 2009;120:2367-76.

24. Mestas $\mathbf{J}$, Hughes CC. Of mice and not men: differences between mouse and human immunology. J Immunol 2004;172:2731-8.

25. Jones SA, Richards PJ, Scheller J, et al. IL-6 transsignaling: the in vivo consequences. J Interferon Cytokine Res 2005;25:241-53.

26. Müllberg J, Schooltink H, Stoyan T, et al. Protein kinase C activity is rate limiting for shedding of the interleukin-6 receptor. Biochem Biophys Res Commun 1992; 189:794-800

27. Müllberg J, Schooltink H, Stoyan T, et al. The soluble interleukin-6 receptor is generated by shedding. Eur $J$ Immunol 1993;23:473-80.

28. Lust JA, Donovan KA, Kline MP, et al. Isolation of an mRNA encoding a soluble form of the human interleukin-6 receptor. Cytokine 1992;4:96-100.

29. Horiuchi S, Koyanagi Y, Zhou Y, et al. Soluble interleukin-6 receptors released from T cell or granulocyte/macrophage cell lines and human peripheral blood mononuclear cells are generated through an alternative splicing mechanism. Eur J Immunol 1994;24:1945-8.

30. Bauer J, Bauer TM, Kalb T, et al. Regulation of interleukin 6 receptor expression in human monocytes and monocyte-derived macrophages. Comparison with the expression in human hepatocytes. J Exp Med 1989;170:1537-49.

31. Desgeorges A, Gabay C, Silacci P, et al. Concentrations and origins of soluble interleukin 6 receptor-alpha in serum and synovial fluid. J Rheumatol 1997:24:1510-16.

32. Marin V, Montero-Julian F, Grès S, et al. Chemotactic agents induce IL-6Ralpha shedding from polymorphonuclear cells: involvement of a metalloproteinase of the TNF-alpha-converting enzyme (TACE) type. Eur J Immunol 2002;32:2965-70.

33. Reitamo S, Remitz A, Varga J, et al. Demonstration of interleukin 8 and autoantibodies to interleukin 8 in the serum of patients with systemic sclerosis and related disorders. Arch Dermatol 1993;129:189-93.

34. Furuse S, Fujii H, Kaburagi Y, et al. Serum concentrations of the CXC chemokines interleukin 8 and growth-regulated oncogene-alpha are elevated in patients with systemic sclerosis. J Rheumatol 2003;30:1524-8.

35. Kadono T, Kikuchi K, Ihn H, et al. Increased production of interleukin 6 and interleukin 8 in scleroderma fibroblasts. J Rheumatol 1998;25:296-301.

36. Kowal-Bielecka 0, Kowal K, Distler 0, et al. Cyclooxygenase- and lipoxygenasederived eicosanoids in bronchoalveolar lavage fluid from patients with scleroderma lung disease: an imbalance between proinflammatory and antiinflammatory lipid mediators. Arthritis Rheum 2005;52:3783-91.

37. Distler 0, Pap T, Kowal-Bielecka 0, et al. Overexpression of monocyte chemoattractant protein 1 in systemic sclerosis: role of platelet-derived growth factor and effects on monocyte chemotaxis and collagen synthesis. Arthritis Rheum 2001:44:2665-78.

38. Chalaris A, Rabe B, Paliga $K$, et al. Apoptosis is a natural stimulus of IL6R shedding and contributes to the proinflammatory trans-signaling function of neutrophils. Blood 2007; 110:1748-55

39. Kaplanski G, Marin V, Montero-Julian F, et al. IL-6: a regulator of the transition from neutrophil to monocyte recruitment during inflammation. Trends Immunol 2003:24:25-9.

40. Hasegawa M, Sato S, Ihn H, et al. Enhanced production of interleukin-6 (IL-6), oncostatin $\mathrm{M}$ and soluble IL-6 receptor by cultured peripheral blood mononuclear cells from patients with systemic sclerosis. Rheumatology 1999;38:612-17.

41. Feghali CA, Bost KL, Boulware DW, et al. Mechanisms of pathogenesis in scleroderma. I. Overproduction of interleukin 6 by fibroblasts cultured from affected skin sites of patients with scleroderma. J Rheumatol 1992;19:1207-11.

42. Hurst SM, Wilkinson TS, McLoughlin RM, et al. II-6 and its soluble receptor orchestrate a temporal switch in the pattern of leukocyte recruitment seen during acute inflammation. Immunity 2001:14:705-14.

43. McLoughlin RM, Witowski J, Robson RL, et al. Interplay between IFN-gamma and IL-6 signaling governs neutrophil trafficking and apoptosis during acute inflammation. $J$ Clin Invest 2003:112:598-607.

44. Kawaguchi Y, Hara M, Wright TM. Endogenous IL-1alpha from systemic sclerosis fibroblasts induces IL-6 and PDGF-A. J Clin Invest 1999;103:1253-60. 